\title{
Ueber die electromotorischen Kräfte der Froschhaut, ihren Sitz und ihre Bedeutung für die Secretion.
}

\author{
Vorläufige Mittheilung. \\ Von \\ Th. W, Engelmann.
}

Aus den Untersuchungen du Bois-Reymonds und seiner Schüler hat sich ergeben, dass die Hautströme der Frösche, wie der Amphibien überhaupt, electromotorischen Kräften ihren Ursprung verdanken, welche in der Drüsenschicht der Haut ihren Sitz haben. Auch an drüsenreichen Schleimhäuten wurden bekanntlich regelmässige electromotorische Wirkungen nachgewiesen. Da man dieselben in keinem der untersuchten Fälle auf Muskel- oder Nervenströme zurückführen konnte, betrachtete man die secernirenden Eiemente als Sitz der genannten Kräfte. Es lag nahe, eine Bedeutung dieser neuen Art thierisch-electrischer Ströme, der Drüsenströme, für den Process der Secretion zu vermuthen. Schon mehrmals war bemerkt worden, dass mit Hilfe der electrischen Osmose viele der bisher dunkeln Absonderungsvorgänge sich einfach erklären lassen würden. Aber man wurde bereits dadurch von einer weiteren Verfolgung dieses Gedankens abgehalten, weil die Richtung der abgeleiteten Drüsenströme innerhalb der Haut stets gerade die entgegengesetzte von der war, in welcher der Flüssigkeitsstrom während der Secretion sich durch die Haut bewegte.

Die Entdeckung der Contractilität aller Hautdrüsen des Frosches und der Nachweis einer besonderen Schicht contractiler Zellen in dem Epithel der genannten Drüsen (vgl. diess. Archiv Bd. IV.pag. 1) legten die Vermuthung nahe, dass diese contractilen Zellen die hauptsächliche, wo nicht einzige Quelle der electromotorischen Kräfte der Froschhaut sein möchten. Um diese Vermuthung zu prüfen, beobachtete ich mittelst des Mikroskopes die Bewegungserscheinungen, welche an den contractilen Drüsenzellen, bei chemischer, thermischer, electrischer, mechanischer, physiologischer Reizung auftreten und verglich damit die Aenderungen, welche die electromotorische Kraft der Haut durch dieselben Einwirkungen erfährt. Bei den Kraftmessungen befand sich das Präparat in einem kleinen, hermetisch 
abschliessbaren, feuchten Raum, in welchem alle Arten der Reizung aufs Bequemste vorgenommen werden konnten.

Es ergaben sich $u$. a. folgende Thatsachen. Jede Contraction ist von einer vorübergehenden Verminderung der electromotorischen Kraft begleitet. Diese negative Schwankung ist im Allgemeinen um so grösser, je stärker die Contraction. Während einer tetanischen Zusammenziehung der Drüsen, wie sie $\mathfrak{u}$. a. durch sehr kleine Mengen von Kohlensäure, Essigsäure, Ammoniak, durch reinen Wasserstoff oder reines Kohlenoxyd (nach längerem Durchleiten), ferner durch Erwärmung auf etwa $35-40^{\circ} \mathrm{C}$., durch Tetanisiren mit electrischen Strömen, durch intermittirende Reizung der I)rüsennerven u. s. w. hervorgebracht wird, kann die electromotorische Kraft bis auf Null herabsinken und dann nach Beseitigung des Reizes wieder bis zur anfänglichen Höhe steigen. Sie kehrt zurück, bevor die Wiederausdehnung der Drüsen ihren Anfang nimmt. - Unter bestimmten bekannten Umständen steigt nach Ablauf der negativen Schwankung die electromotorische Kraft sehr bedeutend über die anfängliche Höhe. Auch diesem Vorgang entsprechen auffällige mikroskopisch warnehmbare, mechanische Aenderungen der contractilen Drüsenzellen. Diejenigen Einflüsse, welche die Reizbarkeit der Drüsenzellen dauernd vernichten, heben im Allgmeinen auch die electromotorische Wirksamkeit der Haut dauernd auf. Ueberhaupt zeigen die Versuche einen selbst unter ganz specifischen Bedingungen so vollständigen Parallelismus zwischen den mechanischen und electromotorischen Vorgängen, dass es erlaubt ist, die contractile Zellenschicht des Drüsenepithels als die einzige Quelleder Froschhautströme zu betrachten.

Eine nähere Betrachtung und Verfolgung der Erscheinungen im Lichte des vorstehenden Ergebnisses führte alsbald weiter zu der Erkenntniss, dass die electromotorischen Kräfte zur Secretion in unmittelbarster Beziehung stehen. Sie nämlich sind - dieser Schluss erwies sich als unvermeidlich - die Triebkräfte, welche das Secret aus dem Drüsenepithel in die Drüsenhöhle treiben. Mit anderen Worten: Die Absonderung der Hautdrüsen ist nichts Anderes als electrische osmose.

Es lässt sich zunächst zeigen, dass die Richtung der Hautströme innerhalb der Drüsen im Allgemeinen die durch die electrische Osmose verlangte ist, -- trotz des scheinbar widersprechenden Resultats, zu welchem die Versuche mit Ableitung der Haut geführt 
haben. Berücksichtigt man die Form und die räumliche Anordnung der contractilen Zellen innerhalb der Drüsenwand, so ergiebt sich aus der Vertheilung der electrischen Spannungen an der Oberfläche isolirter Hautstücke, dass die contractilen Drüsenzellen in demselben Sinne electromotorisch wirken wie gewöhnliche Muskelfasern, dass zugleich an jeder Drüse die innere Oeffnung des Ausführungsgangs (der abgestutzte obere Pol der kugelförmig gedachten Drüse, um welchen die Enden der contractilen Zellen rosettenförmig herumgelagert sind) als natürlicher Querschnitt, die Oberfläche der Drüse als natürlicher Längsschnitt in electromotorischer Beziehung zu betrachten ist. Hiermit ist aber, wie leicht einzusehen, der Forderung betreffs der Richtung der Ströme genügt und zugleich obiger Widerspruch aufgelöst.

Das [nicht contractile] Drüsenepithel erleidet, namentlich an der dem Lumen zugekehrten Oberfläche, während der Secretion höchst charakteristische, mikroskopisch wahrnehmbare Veränderungen, welche unmittelbar auf osmotische Strömungen in dem durch die Theorie verlangten Sinne deuten und sich auch künstlich an andern Gewebselementen mittelst starker galvanischer Ströme hervorrufen lassen.

Die Secretion (speciell das Austreten von Secret aus dem Epithel ins Lumen der Drüse) geschieht continuirlich, und zwar nicht in Folge der Erregung secretorischer Nerven. Sie dauert, wie auch die electromotorische Wirksamkeit der Haut, nach Durchschneidung, ja nach völliger Degeneration sämmtlicher Drüsennerven fort. Nur die motorischen Nerven der contractilen Drüsenzellen haben Einfluss auf die Secretion: ihre Lrregung wirkt hemmend.

Im Allgemeinen ist die Geschwindigkeit der Absonderung der Grösse der electromotorischen Kraft der Haut proportional.

Die electromotorischen Kräfte zwischen Längs- und Querschnitt der Drüsen sind (im Ruhezustand der contractilen Zellen) ausserordentlich gross. Dies folgt aus den bedeutenden Werthen der Kraft zwischen Oberfläche und Unterfläche (oder Querschnitt) der Haut (häufig bis $1 / 10$ ja $1 / 7$ Daniell), unter Berücksichtigung der Grösse und Zahl, der eigenthümlichen Anordnung und Vertheilung der electromotorisch wirksamen Elemente in der Haut. - In Verband hiermit kann der Secretionsdruck ausserordentlich hoch steigen: bei Verstopfung des Ausführungsgangs dehnen sich die Drüsen, durch fortdauerndes Einströmen von Secret ins Lumen, zu grossen prallen Blasen (Retentionscysten) aus. 
Indem ich für die ausführliche Darstellung und Begründung des Vorstehenden auf eine spätere Mittheilung verweise, will ich nur noch auf die, übrigens sehr augenfällige Bedeutung aufmerksam machen, welche die mitgetheilten Ergebnisse für die Auffassung der thierisch-electrischen Erscheinungen überhaupt und zunächst für die Lehre vom ruhenden Muskelstrom besitzen. Die letztgenannte Lehre wird dadurch im Sinne ihres Urhebers aufs Neue befestigt. Zugleich sind in den vorstehenden Mittheilungen einige Winke zur praktischen Behandlung der Präexistenz-Frage enthalten, insofern einige neue Wege angedeutet sind, auf welchen die Vernichtung der electromotorischen Wirksamkeit der Haut ohne Beleidigung der daunter liegenden Muskeln zu erreichen ist.

Utrecht im Juli 1861. 